\title{
Soutien de la direction d'établissement lorsque survient un problème éthique en période d'insertion professionnelle : exploration de quatre avenues
}

Lise-Anne St-Vincent

Université du Québec à Trois-Rivières
Support by the school administration to resolve ethical problems during professional insertion: Four exploratory avenues

\section{ésumé}

Le présent article met en lumière l'importance du rôle de la direction quant à la préparation de

l'équipe-école, notamment les enseignants en insertion professionnelle, pour résoudre en équipe des problèmes comportant des enjeux éthiques. Dans cet article, quatre avenues sont explorées : I'élaboration

d'un cadre de référence éthique par chacun des membres de l'équipe-école, la création d'un espace propice aux échanges sur les valeurs et les principes, la mise à jour des connaissances du cadre professionnel et enfin la mise en œuvre de procédures de résolution Iorsqu'un problème éthique se présente.

Mots-clés

direction d'établissement, enseignant en insertion professionnelle, résolution de problèmes éthiques, approche dialogique, cadre professionnel

\section{Abstract}

This article sheds light on the administration's critical role in preparing the school team, and particularly teachers in professional insertion, to resolve ethical issues as a team. Four avenues are explored: developing an ethical framework for each school team member, creating a space conducive to sharing values and principles, updating knowledge of the professional framework, and implementing procedures for resolving ethical problems as they arise.

\section{Keywords}

school administration, professional insertion, ethical problem solving, dialogical approach, professional framework

\section{Introduction}

$\mathrm{Au}$ cours des dernières décennies, plusieurs changements exogènes (Baby, 2012) ont modifié l'environnement éducatif québécois (Deniger, 2012; Fournier et Fréchette, 2012; Tardif, 2013) tels que le multiculturalisme, l'intégration des élèves ayant des besoins spécifiques, la mise en place de nouvelles politiques ou encore l'avancée des technologies. Cela a eu pour effet de placer les milieux scolaires dans un état d'instabilité. Pour les enseignants débutants, qui doivent composer avec une multitude de tâches non familières dès l'entrée en pratique, cette réalité complexifie davantage leur insertion professionnelle. Les nombreuses transformations survenues dans les milieux éducatifs supposent, entre autres, une augmentation des interactions entre les divers acteurs scolaires (Trépanier, 2005). Celles-ci se traduisent parfois par des échanges propices à l'apparition de conflits de valeurs (Legault, 2003) ou de principes (StVincent, 2011a), soit des problèmes d'ordre éthique. Il n'existe pas de solutions claires et objectives pour répondre à ce type de problèmes (Clarkeburn, 2002; Cranston, Ehrich et Kimber, 2014; Shapiro, Stefkovich et Gutierrez, 2014). En conséquence, leur résolution s'avère plus complexe que des problèmes liés à la gestion de comportements par exemple, et nécessite un dialogue entre plusieurs individus (Bourgeault, 2012; Mushipu Mbombo, 2011; Patenaude, 1998). Le soutien de la direction d'établissement devient alors un facteur central pour atteindre une conciliation entre les personnes concernées par les enjeux éthiques (Norberg et Johansson, 2007; St-Vincent, 2013b). La direction doit coordonner les démarches individuelles et collectives dans un processus de résolution de problèmes : « Les directions jouent un rôle politique de médiateurs et tentent de 
maintenir la cohésion et l'unité du milieu au moyen de leurs compétences relationnelles » (Poirel et Yvon, 2012, p. 611). Elle doit également s'assurer de la cohérence de l'ensemble des décisions au regard des encadrements légaux et réglementaires, tel que le stipule le programme de formation québécois à la gestion d'un établissement d'enseignement (Ministère de l'Éducation, du Loisir et du Sport [MELS], 2008). De surcroit, les enseignants débutants expriment qu'ils vivent plusieurs craintes devant un problème comportant des enjeux éthiques en raison de leur statut précaire (St-Vincent, 2011a, 2011b). Ces craintes interfèrent parfois dans l'analyse du problème et peuvent ainsi compromettre sa résolution. Par conséquent, les préoccupations personnelles de l'enseignant peuvent voiler les enjeux réels dans une situation complexe (St-Vincent, 2011a). Cela amène une autre difficulté : un problème d'ordre éthique qui n'est pas résolu ou encore mal résolu risque de placer les enseignants dans une posture encore plus compliquée qu'initialement. Les enseignants en insertion professionnelle manifestent un besoin de repères et d'accompagnement dans leur développement professionnel (Gervais, 1999; Mukamurera, Portelance et Martineau, 2015) et la direction d'établissement peut jouer un rôle important dans ces situations. Elle peut, entre autres, faciliter une prise de distance nécessaire devant l'ensemble des enjeux qui se présentent avant que l'enseignant ne s'engage de manière précipitée dans la résolution d'un problème éthique.

Mobiliser des ressources rapidement en réaction aux problèmes comportant des enjeux éthiques qui se présentent peut parfois s'avérer nécessaire afin d'éviter l'aggravation d'un problème. Toutefois, il demeure impératif que la direction d'établissement prenne aussi le temps de s'interroger sur les fondements qui sous-tendent ses propres décisions et ses agissements : « [Le directeur d'établissement scolaire] incarne un modèle. Il se tourne et donne l'exemple en assumant tout à la fois les dimensions du droit, de la morale et de l'éthique, ainsi que les tensions engendrées par leur coexistence » (Obin, 1996, p. 152). La direction d'établissement soucieuse d'accompagner adéquatement les enseignants en insertion professionnelle doit donc au préalable faire l'exercice de cerner son propre cadre de référence éthique. Ensuite, à titre de gestionnaire, elle doit faire l'examen des besoins du milieu et des structures en place pour soutenir l'ensemble des membres de l'équipe-école lorsque survient ce type de problèmes, particulièrement en ce qui concerne les enseignants en insertion professionnelle. Finalement, elle doit faciliter la mobilisation des ressources lorsque vient le moment de les résoudre.

Dans cet article, nous portons un regard particulier sur l'accompagnement des enseignants en insertion professionnelle devant un problème d'ordre éthique. Nous approfondissons certains éléments qui peuvent favoriser une meilleure préparation de l'enseignant et lui assurer le soutien nécessaire. Pour ce faire, nous explorons quatre avenues : la création d'un espace propice à la communication des valeurs et des principes des différents acteurs scolaires qui forment la communauté, le développement du cadre de référence éthique des membres de l'équipe-école, la mise à jour des connaissances du cadre professionnel des différents intervenants ainsi que la mise en œuvre de procédures lorsque survient le problème. Cependant, avant d'explorer ces quatre avenues, il est essentiel de déterminer ce qu'est un problème éthique et de quelle manière les enseignants en insertion professionnelle ont tendance à appréhender un tel type de problème. 


\section{Reconnaitre un problème éthique}

Tout problème rencontré dans les milieux d'éducation n'est pas d'emblée ce que nous identifions comme un problème d'ordre éthique. Un problème comportant des enjeux éthiques réfère avant tout à une situation complexe dans laquelle plusieurs personnes sont impliquées (Legault, 2003). Ce sont les divers points de vue auxquels celles-ci adhèrent qui font émerger des conflits de valeurs ou de principes (St-Vincent, 2011a). Chaque individu possède ses propres référents qui sous-tendent ses décisions : valeurs personnelles, principes professionnels et certains éléments connus des règles ou des lois. Lorsqu'un problème éthique survient, des décisions doivent être prises et des actions doivent être mises en œuvre pour modifier la situation. Plusieurs éléments doivent alors être considérés et leur importance déterminée (Cranston et al., 2014; Legault, 2003). Par conséquent, le processus de résolution présente un caractère subjectif (Clarkeburn, 2002).

À titre d'exemple, Julie, une enseignante débutante, prend l'initiative d'aller rencontrer un parent à la maison avec l'intention d'aider un jeune qui s'absente souvent, et ceci, sans en aviser la direction. Au bout d'un moment, le parent s'agite et lui ordonne de partir, en lui disant qu'elle « ne se mêle pas de ses affaires ". À la suite de cette visite, le parent porte plainte à la direction d'établissement et à la commission scolaire en déformant les paroles de l'enseignante et en leur prêtant des intentions d'ingérence. La direction peut ici s'interroger quant aux gestes à poser : expliquer à l'enseignante qu'elle doit être prudente et discuter avec elle de son rôle professionnel? Donner raison au parent? Protéger la réputation de l'enseignante auprès des membres de la commission scolaire? L'encourager à rencontrer les parents pour clarifier la situation? Cette absence de réponses claires qui génère de l'incertitude et de l'inconfort devient un indice important pour reconnaitre un problème d'ordre éthique.

\section{L'appréhension des problèmes éthiques par des enseignants débutants}

Devant des problèmes comportant des enjeux éthiques, les enseignants en insertion professionnelle peuvent rapidement ressentir que leurs convictions morales sont insuffisantes (Moreau, 2003). Dans nos travaux de recherche auprès de novices en enseignement au Québec, nous avons fait ressortir certaines de leurs préoccupations exprimées quant à la fiabilité de leur propre jugement professionnel lorsque se présente un problème éthique, aussi en ce qui a trait à l'incompréhension de certains de leurs rôles dans ces situations (St-Vincent, 2011a, 2011b). Par ailleurs, les enseignants débutants mentionnent des incertitudes en ce qui a trait à la collégialité, qui réfère au sentiment d'appartenance à l'équipe-école et au besoin de s'intégrer au nouvel environnement (Gervais, 1999). En ce sens, plusieurs des inquiétudes sont liées étroitement au statut précaire (Belzile, 2008; Mukamurera et Gingras, 2005; Martineau et Presseau, 2003). Il faut rappeler qu'il y a actuellement près de $40 \%$ des enseignants qui vivent des situations de précarité au Québec (Tardif, 2013) :

les enseignants précaires héritent bien souvent du « sale boulot »: des classes plus difficiles, une tâche éclatée nécessitant, par exemple, plusieurs préparations pour des cours différents, une charge de travail répartie entre plusieurs écoles, une intégration déficiente dans les établissements, une faible reconnaissance dans les milieux, etc. (p. 235) 
Toutes ces préoccupations relatives à l'insertion professionnelle peuvent biaiser l'analyse d'une situation et la reconnaissance d'un problème éthique. Voici un exemple illustrant une telle situation : Olivier, un enseignant débutant, se lie d'amitié avec une collègue. Cette dernière l'amène dans une soirée chez des amis où il se rend compte de la présence de quelques élèves de l'école consommant de la marijuana sur place. Olivier peut vivre un grand malaise et être profondément troublé face à cette situation, mais refuser de dénoncer ce fait par crainte de perdre une alliance significative dans l'école ou encore de ternir sa réputation dans la communauté enseignante. De surcroit, un problème éthique non résolu ou mal résolu risque de se complexifier. Ainsi, dans l'exemple évoqué, si Olivier se tait et qu'un élève raconte cette soirée à son parent, la situation pourrait s'aggraver rapidement.

Considérant ce phénomène, il semble important qu'un enseignant novice soit accompagné afin de l'aider à distinguer entre les préoccupations relatives à son insertion professionnelle et les enjeux concernant le problème éthique lui-même. Ceci, afin d'éviter la confusion dans sa réflexion et la mise en œuvre d'actions qui ne répondraient pas au problème éthique rencontré, mais plutôt à un souci lié à son insertion professionnelle.

Par ailleurs, la direction d'établissement se retrouve souvent seule pour prendre la décision finale devant un problème comportant des enjeux éthiques (St-Vincent, 2013a). Pourtant, les interrogations soulevées à l'examen du problème incitent à la réflexion collective et au dialogue. Il semble judicieux que ce processus ne s'improvise pas. Une préparation de l'ensemble de tous les membres de l'équipe-école devient alors incontournable. Celle-ci peut s'inscrire dans les quatre avenues proposées : l'élaboration d'un cadre de référence éthique, la création d'un espace propice aux échanges sur les valeurs et les principes, la mise à jour des connaissances du cadre professionnel et la mise en œuvre de procédures de résolution.

\section{Première avenue : élaboration d'un cadre de référence éthique pour l'équipe-école}

Dans leurs recherches effectuées en Pennsylvanie, aux États-Unis, Shapiro et Stefkovich (2011) font ressortir l'importance de l'élaboration d'un cadre de référence éthique par les différents professionnels des milieux éducatifs. Cet exercice vise à prendre conscience des valeurs, des principes et des éléments du cadre professionnel sous-jacents à ses décisions et ses agissements. Toujours en Pennsylvanie aux États-Unis, les travaux de Tuana (2014) désignent ce cadre de référence comme la littératie morale d'un individu, celle-ci étant constituée de trois éléments : la sensibilité éthique, les compétences pour prendre des décisions éthiques et la motivation éthique (p. 154). Sous cet angle, le cadre de référence éthique ou encore la littératie morale suppose un processus, un développement. Ainsi, devant l'incertitude, les enseignants vont faire certains choix, souvent en référence à des valeurs personnelles (Husu et Tirri, 2001), bien qu'ils n'en soient pas toujours conscients (Jackson, Boostrom et Hansen, 1993). Un exercice de mise en situation sous forme de vignettes écrites (St-Vincent, 2013b) peut aider chaque intervenant à identifier et définir les principes professionnels auxquels il adhère et mettre en évidence les valeurs qu'il souhaite actualiser (Fortin et Parent, 2004), c'est-à-dire ce qui a de l'importance pour lui. Il doit aussi cerner les éléments qu'il connait et qui font partie de son cadre professionnel. À titre d'exemple, il est possible de présenter une vignette qui décrit le cas d'Olivier mentionné plus haut et poser les questions suivantes : quels sont les enjeux éthiques selon vous dans cette situation et quelles actions envisageriez-vous et pourquoi? L'enseignant en insertion professionnelle aura tendance à avoir 
une réflexion basée davantage sur des idéaux, ayant trop peu d'expérience en tant que professionnel pour avoir une posture claire, parfois oscillant encore entre le statut d'étudiant et d'enseignant (Baillauquès, 1999). Cependant, c'est à travers cette réflexion initiale qu'il aura l'occasion d'exprimer sa vision de la pratique professionnelle. Le rôle de la direction d'établissement n'est pas de provoquer une désillusion chez l'enseignant à cette étape, ce qui survient naturellement dans le processus d'insertion (Mukamurera, Martineau, Bouthiette et Ndoreraho, 2013), mais plutôt de l'aider à reconnaitre les structures sous-jacentes de ses décisions actuelles. Ce type d'activité peut s'avérer un déclencheur pour amorcer des débats de fond. À cet effet, il est souhaitable de créer un espace physique et temporel propice aux échanges sur les valeurs et les principes professionnels afin d'alimenter la réflexion et non de faire naitre ou d'amplifier des divergences entre les membres de l'équipe-école.

\section{Deuxième avenue : création d'un espace propice aux échanges sur les valeurs et les principes}

La valeur d'un cadre de référence éthique se manifeste lors des échanges professionnels. Il devient utile de mieux comprendre les interventions de chacun lorsque les interlocuteurs justifient leur position en s'appuyant sur leurs repères. Toutefois, les activités communicationnelles de cet ordre demeurent constructives si certaines conditions sont respectées. Dans leurs recherches sur le développement du souci éthique dans les pratiques professionnelles au Québec, Fortin et Parent (2004) se sont intéressés aux groupes de réflexion et de discussion où la parole est possible. Ainsi, ils relèvent dans leurs travaux plusieurs conditions qui favorisent le développement du savoir sur sa pratique professionnelle et le questionnement sur les enjeux éthiques qu'elle soulève. Entre autres :

Les groupes de réflexion trouvent toute leur pertinence lorsqu'ils : ne sont pas ramenés à des groupes dont la finalité est uniquement d'ordre technique [...]; parviennent à garantir un climat de confiance, d'écoute et de parole de l'autre, où les réussites, les limites ou les échecs de chacun sont accueillis avec respect; contribuent à guider la réflexion éthique au sein de l'établissement; permettent d'humaniser encore davantage les pratiques [...] (p. 262)

De tels espaces de rencontre peuvent être d'abord initiés par un professionnel hors de l'équipe-école, pour être animés ensuite par un membre nommé par les autres, sans lien hiérarchique (Fortin et Parent, 2004; Fortin, Leclerc, Parent, Plourde et Rondeau, 2011). La participation doit s'effectuer sur une base volontaire. C'est l'occasion d'exprimer des malaises et des interrogations devant des situations ambigües. Voici quelques exemples : « jusqu'où s'applique le principe de confidentialité avec le jeune? »; « je me sens mal lorsqu'on me demande d'évaluer un jeune qui ne peut progresser au même rythme que les autres, j'ai l'impression que ce n'est pas juste pour lui ». Dans leur recherche, Fortin et al. (2011) expliquent que ces inconforts sont des éléments déclencheurs dans la réflexion : «Loin de paralyser la réflexion, il [le malaise] la met en action. Le fait ensuite de dénoncer et de nommer ce qu'on dénonce semble être le début d'une réflexion, d'un travail d'intelligence, d'un travail de la pensée.» (p. 60) Dans le doute, lenseignant en insertion professionnelle exprime le besoin de se confier, de partager ses émotions et la direction n'est pas toujours disponible ou encore n'est pas la personne ressource adéquate pour ce soutien (St-Vincent, 2011a, 2011b). Ces espaces deviennent ainsi un endroit adéquat pour l'aider à construire son identité professionnelle. 


\section{Troisième avenue : mise à jour des connaissances du cadre professionnel des différents intervenants}

Les politiques évoluent rapidement et il devient difficile de tenir le rythme comme professionnels. La direction d'établissement est souvent désignée comme la gardienne de ce cadre légal et réglementaire (MELS, 2008) dans son établissement. Nous avons mis en évidence dans nos travaux la méconnaissance du cadre professionnel chez les enseignantes débutantes (St-Vincent, 2011a, 2011b, 2013c). En effet, le cadre professionnel est souvent considéré par les novices comme une entité de l'ordre des contraintes, de la reddition de compte et des recours extérieurs et non comme un mécanisme de soutien et de repères, guidant les actions. Cependant, l'ignorance de certains éléments du cadre peut mener à des prises de décisions ou des actions lourdes de conséquences, tel que des études effectuées au Québec le mettent en évidence (Jeffrey, 2011; Jeffrey, Deschênes, Harvengt et Vachon, 2009). Bien que les enseignants québécois ne fassent pas partie d'un ordre professionnel actuellement et qu'ils ne se réfèrent pas à un code de déontologie, « différents documents ministériels peuvent néanmoins fournir certaines balises dans l'adoption d'agir éthique "(Cloutier, St-Vincent et Loiola, 2015). En Ontario, les enseignants font partie d'un ordre, et la connaissance des normes s'avère essentielle, car les dilemmes rencontrés font souvent émerger certaines contradictions dans leur application, tel que le font ressortir les études de Campbell (2003).

Reprenons l'exemple de Julie. Sait-elle où s'arrête le rôle de l'enseignant et où commence celui des parents selon la loi? A-t-elle le droit de se présenter à la résidence d'un élève, et de plus, sans en aviser la direction? Que dit la convention des enseignants lorsqu'il y a une plainte officielle? Quelles sont les obligations de la direction envers les parents? Les enseignants? La commission scolaire?

Devant un problème comportant des enjeux éthiques, l'application des normes, qu'elles soient internes ou externes, ne va pas de soi (Fortin et al., 2011). Parfois même, certaines se contredisent (Bourgeault, 2012). La direction d'établissement se retrouve généralement la première personne « coincée » entre ces contradictions.

C'est pourquoi il est fondamental qu'une mise à jour des connaissances des règlements internes, des conventions collectives, des règlements administratifs et de certaines lois soit effectuée régulièrement auprès des membres de l'équipe-école et que les enseignants en insertion professionnelle soient présents. Cette activité, enrichie de cas concrets, peut devenir un moteur important dans les échanges et un incitatif à mettre en œuvre des procédures de résolution de problèmes éthiques en équipe.

\section{Quatrième avenue : mise en œuvre de procédures de résolution}

La mise en œuvre de procédures de résolution vise à empêcher des prises de décisions trop rapides pour régler un problème complexe. Les décisions spontanées prises devant un problème éthique ne résultent pas d'une réflexion critique, mais plutôt d'une réponse impulsive pour régler une situation souvent inconfortable. En effet, ces décisions ont peu de chance de tenir compte de tous les éléments importants ou des différentes perspectives des personnes impliquées. Le dialogue se présente alors comme un réel exercice éthique, soit une production conjointe de sens (Patenaude, 1998). À cet effet, Legault (2003) propose une délibération dialogique dans un processus de construction de solution intéressant : 
La prise de décision est un raisonnement pratique en éthique dans la mesure où elle vise à trouver « la meilleure solution possible dans les circonstances ». L'approche dialogique parce que la «meilleure solution » n'est pas la meilleure pour moi, mais pour « nous », mais pas seulement le «nous » du groupe ou de cette communauté, mais le «nous » des personnes qui désirent envisager la solution d'un point de vue raisonnable.» (p. 245)

Certains modèles sont proposés dans la littérature pour résoudre des dilemmes éthiques. Dans leurs travaux réalisés en Australie, Cranston et al. (2014) proposent un modèle amenant à analyser et résoudre le problème à travers différentes composantes : l'incident critique, les facteurs à considérer, les valeurs et les croyances de l'individu, le choix dans les décisions possibles et les implications. Ce modèle traite $\mathrm{du}$ processus de résolution de la direction, mais n'explique pas clairement la contribution des autres acteurs scolaires. Par ailleurs, en Pennsylvanie, aux États-Unis, Tuana (2014) relève aussi une série d'actions permettant de prendre une décision éthique : la considération des éléments pertinents des cadres de référence (les conséquences des actions, les droits, les obligations, les relations à préserver); la prise en compte de certains éléments du contexte; l'identification des personnes impliquées et leurs valeurs; l'évaluation et la mise en œuvre des actions. Bien que ce modèle présente des lignes d'action plus précises, le canevas demeure difficile à opérationnaliser. Au Québec, en 2003, Legault a proposé un modèle pour résoudre un problème éthique qui comporte quatre phases : prendre conscience de la situation, clarifier les valeurs conflictuelles, prendre une décision éthique et établir un dialogue réel entre les personnes impliquées. Cela suppose que l'ensemble des membres de l'équipe-école s'engage dans un processus de réflexion collective lorsqu'un problème éthique semble émerger. La grille de délibération proposée par l'auteur peut s'avérer fastidieuse à respecter dans un milieu dans lequel la simultanéité et la multiplicité des tâches surchargent les horaires, car elle comprend plusieurs étapes et nécessite un certain entrainement. Cependant, ce modèle met en exergue certains repères importants pouvant guider une équipe école pour décider des procédures à mettre en œuvre lorsqu'un problème éthique émerge :

- inventorier les principaux éléments de la situation, dont la dimension normative;

- $\quad$ formuler le dilemme;

- $\quad$ identifier les conflits de valeurs ou de principes;

- $\quad$ identifier les valeurs ou les principes qui ont préséance et pourquoi;

- $\quad$ préciser les modalités des actions;

- formuler une argumentation et une justification de la position.

«Inventorier les principaux éléments de la situation » implique au départ que les membres de l'équipeécole développent une pratique de conservation de traces : dossiers d'élèves, observations en classe ou dans le cadre de diverses activités à l'école, travaux significatifs, brefs résumés de conversations téléphoniques, correspondances électroniques, résumés de faits importants, etc. Ensuite, il s'agit de reconstituer une chronologie dans les évènements. Aussi, il est important de relever tous les constituants de la dimension normative liés à la situation: politiques, règles, loi ou autres. Finalement, il est important de mentionner qu'il faudra décider à travers cet inventaire des personnes devant faire partie des échanges, selon le problème et le contexte. Pour un enseignant en insertion professionnelle, il est 
possible qu'il n'ait pas développé ces gestes de « conservation des traces ». Comme il est particulièrement susceptible d'être confronté à des problèmes éthiques dès son entrée dans le milieu, une explication de l'importance de ces automatismes peut s'avérer utile.

«Formuler le dilemme " consiste à déterminer le problème central à résoudre. Dans les milieux éducatifs, le problème central se présente comme un choix à faire entre une série de principes qui entrent en compétition, et ce, souvent dans un contexte complexe empreint de valeurs (Cranston et al., 2014). Selon Malherbe (2000), un dilemme se pose comme deux plans d'action contradictoires. Ainsi, dans le problème évoqué initialement dans lequel l'enseignante Julie rend visite aux parents et que ceuxci portent plainte, il est possible d'en arriver à un dilemme comme celui-ci : « considérant plusieurs éléments tels que le fait que l'enseignante n'ait pas avisé la direction de cette visite à domicile au départ, de la loi par rapport au harcèlement, qu'une plainte ait été déposée, mais aussi de la bienveillance de son intervention et de son ouverture au dialogue, l'enseignante doit-elle rencontrer ou non les parents pour discuter de ces évènements et tenter de régler la situation? ". Si l'enseignante choisit de régler la situation directement avec les parents, elle pourra justifier ses actes et peut-être ne pas exposer l'élève à plus de difficultés qu'initialement, même trouver de nouvelles solutions. Par contre, si certains principes légaux ne sont pas respectés, il est possible que ce choix entraine d'autres conséquences plus lourdes pour elle et pour la direction d'établissement. L'enseignante au centre des conflits aura de la difficulté à cerner les enjeux éthiques. De plus, un enseignant débutant aura peut-être des préoccupations supplémentaires liées à son insertion professionnelle, par exemple « compromettre sa crédibilité auprès des collègues ou réduire ses chances d'obtenir un nouveau contrat ", et c'est à cette étape qu'une réflexion collective devient cruciale et que la direction d'établissement peut apporter des éclairages déterminants.

"Identifier les conflits de valeurs ou de principes en jeu » met en évidence l'apport principal de l'élaboration d'un cadre de référence éthique expliqué dans la première avenue. Un enseignant en insertion professionnelle n'aura peut-être pas eu la chance de participer à ces activités lui permettant d'élaborer avec les autres membres son cadre de référence éthique, mais une réflexion écrite, dès son entrée en fonction, discutée ensuite dans un espace propice, comme il est proposé dans la deuxième avenue s'avèrerait bénéfique pour lui. Ses collègues auraient alors l'occasion de connaitre les structures qui sous-tendent ses décisions professionnelles et de réaffirmer les leurs.

«Identifier les valeurs et les principes ayant préséance » est un exercice qui exige de respecter certaines règles de base dans une activité communicationnelle. Chaque participant doit être en mesure d'exprimer ce qu'il juge le plus important dans la situation. Les interlocuteurs doivent s'exprimer et s'écouter dans un climat respectueux afin de décider ensemble de la prépondérance des éléments dans la situation. Un enseignant en insertion professionnelle pourra se sentir plus en sécurité dans un climat d'écoute déjà établi, dans lequel il est prévu qu'il s'exprime au même titre que les autres intervenants.

"Préciser les modalités d'action" signifie d'effectuer l'inventaire des moyens et des stratégies qui répondent le mieux aux valeurs ou principes déterminés prépondérants. Cela exige une certaine connaissance des ressources disponibles, mais de la créativité également. Si l'enseignant en insertion professionnelle ne connait pas toutes les ressources disponibles et les contraintes existantes, il peut toutefois émettre des idées nouvelles et sa contribution peut s'avérer très enrichissante. 
« Formuler une argumentation et une justification de la position » est une étape dans laquelle l'expérience de la direction d'établissement et sa connaissance du milieu deviennent précieuses. L'ensemble des idées émises par le groupe de délibération peut servir à la rédaction d'un rapport explicatif. Ici, l'enseignant débutant apprendra certaines règles du cadre professionnel en discutant avec ses collègues. La direction d'établissement doit rendre des comptes à ses supérieurs et présenter une justification des décisions prises avec une argumentation recevable par la commission scolaire et les autorités. Cependant, par cette procédure, elle n'est pas seule à prendre la décision finale, même si elle en devient la représentante ainsi que la gardienne des actions qui y sont liées.

\section{Conclusion}

Les débuts de la pratique en enseignement sont empreints de plusieurs adaptations. Les milieux éducatifs, eux-mêmes, sont sujets à des changements constants. Les problèmes comportant des enjeux éthiques sont maintenant plus susceptibles d'apparaitre de manière inattendue en raison de la croissance des interactions entre les nombreux acteurs scolaires. Devant ces situations complexes, les enseignants en insertion professionnelle ont besoin d'un soutien adéquat pour prendre des décisions, et ce, particulièrement de la part de la direction d'établissement.

De plus, il est possible que les enseignants débutants, en raison de leur statut précaire entre autres, présentent des préoccupations supplémentaires relatives à l'insertion professionnelle, ce qui empêche un examen clair des enjeux éthiques présents dans le problème. Certes, la direction d'établissement peut intervenir ponctuellement auprès des enseignants pour les aider à prendre du recul, mais elle peut également adopter une approche proactive et préparer l'ensemble de son équipe-école. En ce sens, quatre avenues ont été présentées : l'élaboration d'un cadre de référence éthique par chacun des membres à l'aide d'activités de réflexion, la création d'un espace propice aux échanges sur les valeurs et les principes en respectant des règles de communication, la mise à jour des connaissances du cadre professionnel et la mise en œuvre de procédures de résolution lorsqu'un problème éthique se présente. Ces avenues représentent des repères pour aider les directions d'établissement à dresser un plan d'action à moyen ou long terme.

Les enseignants en insertion professionnelle s'intégreront plus facilement dans un milieu où les échanges se font dans un climat respectueux et dans lequel les activités de réflexions et l'approche dialogique font partie de la culture des pratiques. Afin de favoriser un environnement de cette nature, la direction d'établissement doit au départ se considérer comme un modèle et prendre le temps de s'interroger. De plus, elle doit miser sur l'écoute et instaurer la mise en œuvre des moyens menant à un travail de résolution de problèmes éthiques en équipe.

Le rythme accéléré des milieux scolaires incite peu à l'aménagement d'une plage horaire pour les échanges et la réflexion. Cependant, des projets semblables, explorés dans certaines recherches au Québec (Fortin et al., 2011; St-Vincent, 2013b), font ressortir un sentiment de soulagement exprimé chez les professionnels, leur permettant de sortir de l'isolement dans les moments difficiles.

Ce sont les retombées à long terme de telles initiatives qui permettront de démontrer en quoi ce type d'engagement envers l'équipe-école a le potentiel d'instituer une certaine stabilité et une résilience devant des problèmes éthiques dans un milieu éducatif. 


\section{Références}

Baby, A. (2012). L'école et son rapport au changement. Dans N. Rousseau (dir.), Modèle dynamique de changement accompagné en contexte scolaire : pour le bien-être et la réussite de tous (p. 29-46). Québec: Presses de l'Université du Québec.

Baillauquès, S. (1999). Ce que l'entrée dans la carrière révèle du rapport des enseignants et des enseignantes à la formation : éléments d'une problèmatique de professionnalisation. Dans J.-C. Hétu, M. Lavoie et S. Baillauquès (dir.), Jeunes enseignants et insertion professionnelle : un processus de socialisation? De professionnalisation? De transformation? (p. 6283). Paris : De Boeck.

Belzile, M. (2008). Étude de la manifestation du souci éthique dans la pratique éducative d'enseignantes du primaire en début de carrière. (Mémoire de maîtrise, Université du Québec à Rimouski, Rimouski, Québec). Repéré à http://semaphore.uqar.ca/104/1/Melanie Belzile decembre2008.pdf

Bourgeault, G. (2012). Éthique professionnelle et réflexivité : quelle connivence? Dans M. Tardif, C. Borges et A. Malo (dir.), Le virage réflexif en éducation : où en sommes-nous 30 ans après Shön? (p. 107-119). Bruxelles : De Boeck. http://dx.doi.org/10.3917/dbu.tardi.2012.01.0107

Campbell, E. (2003). Let right be done : Trying to put ethical standards into practice. Dans P. T. Begley et O. Johansson (dir.), The Ethical Dimensions of School Leadership (p. 107-125). Pays-Bas : Springer. http://dx.doi.org/10.1007/0-306-48203-7 7

Cloutier, G., St-Vincent, L.-A. et Loiola, F. (2015). L'émergence de préoccupations éthiques dans les programmes de formation initiale à l'enseignement. Dans L.-A. St-Vincent (dir.), Le développement de l'agir éthique chez les professionnels en éducation: Formations initiale et continue (p. 5-23). Québec, QC : Presses de l'Université du Québec.

Clarkeburn, H. (2002). A test for ethical sensitivity in science. Journal of Moral Education, 31(4), 439-453. http://dx.doi.org/10.1080/0305724022000029662

Cranston, N., Ehrich, L. C. et Kimber, M. (2014). Managing ethical dilemmas. Dans C. M. Branson et S. J. Gross (dir.), Handbook of Ethical Educational Leadership (2e éd.) (p. 229-245). New York, NY : Routledge. http://dx.doi.org/10.4324/9780203747582.ch15

Deniger, M.-A. (2012). La gestion et l'appropriation du changement en éducation. Éducation et francophonie Éducation et francophonie, $X L(1), 1-11$. http://www.acelf.ca/c/revue/sommaire.php?id=35\#.VpUknpPhBE4

Fortin, P. et Parent, P.-P. (dir.) (2004). Le souci éthique dans les pratiques professionnelles. Paris : L'Harmattan. http://dx.doi.org/10.3917/har.forti.2004.01

Fortin, P., Leclerc, B., Parent, P.-P., Plourde, S. et Rondeau, D. (2011). La Construction du savoir éthique dans les pratiques professionnelles. Paris : L'Harmattan. http://dx.doi.org/10.3917/har.coll.2011.01

Fournier, H. et Fréchette, S. (2012). Vers une prise en compte de la complexité du changement en contexte scolaire québécois : une synthèse des publications. Dans N. Rousseau (dir.), Modèle dynamique de changement accompagné en contexte scolaire : pour le bien-être et la réussite de tous (p. 47-65). Québec: Presses de l'Université du Québec.

Gervais, C. (1999). Analyse du discours d'enseignants sur des pratiques d'accompagnement en insertion professionnelle : une pratique d'accompagnement à l'image de la pratique d'enseignement. Dans J.-C. Hétu, M. Lavoie et S. Baillauquès (dir.), Jeunes enseignants et insertion professionnelle : un processus de socialisation? De professionnalisation? De transformation? (p. 114-133). Paris : De Boeck.

Husu, J., et Tirri, K. (2001). Teachers' ethical choices in socio-moral settings. Journal of Moral Education, 30(4), 361-375. http://dx.doi.org/10.1080/03057240120094850

Jackson, P. W., Boostrom, R. E. et Hansen, D. T. (1993). The moral life of schools. San Francisco, CA : Jossey-Bass.

Jeffrey, D. (2011). L'intervention physique, qu'en penser? Formation et profession, 18(1), 26-29. Repéré à http://www.crifpe.ca/download/verify/1121

Jeffrey, D., Deschênes, G., Harvengt, D. et Vachon, M.-C. (2009). Le droit et l'ethique. Dans F. Jutras et C. Gohier (dir.), Repères pour l'éthique professionnelle des enseignants (p. 75-91). Québec, QC : Presses de l'Université du Québec. 
Legault, G. A. (2003). Professionnalisme et délibération éthique. Québec, QC : Presses de l'Université du Québec.

Malherbe, J.-F. (dir.) (2000). Compromis, dilemmes et paradoxes en éthique clinique. Montréal, QC : Artel-Fides.

Martineau, S. et Presseau, A. (2003). Le sentiment d'incompétence pédagogique des enseignants en début de carrière et le soutien à l'insertion professionnelle. Brock Education, 12(2), 54-67. Repéré à

https://brock.scholarsportal.info/journals/brocked/home/article/view/37

Ministère de l'Éducation, du Loisir et du Sport (MELS). (2008). La formation à la gestion d'un établissement d'enseignement. Les orientations et les compétences professionnelles. Repéré à http://www.education.gouv.qc.ca/fileadmin/site web/documents/reseau/formation titularisation/07-00881.pdf

Moreau, D. (2003). La construction de l'éthique professionnelle des enseignants : la genèse d'une éthique appliquée de l'éducation (Doctorat), Université de Nantes, France.

Mukamurera, J., Martineau, S., Bouthiette, M. et Ndoreraho, J.-P. (2013). Les programmes d'insertion professionnelle des enseignants dans les commissions scolaires du Québec : portrait et appréciation des acteurs. Education Eं Formation, (e-299). Repéré à https://www.researchgate.net/profile/Joachim De Stercke/publication/260577616 Insertion professionnelle des enseignants dbutants en francophonie/links/0a85e53787b8dca2a3000000.pdf\#page=13

Mukamurera, J., Portelance, L. et Martineau S. (2015). Développement et persévérance professionnels : Enjeux pour la profession enseignante et l'efficacité des systèmes éducatifs. Dans J. Mukamurera, L. Portelance, et S. Martineau (dir.), Développement et persévérance professionnels : Oui, mais comment? (p. 1-7). Québec, QC : Presses de l'Université du Québec.

Mukamurera, J. et Gingras, C. (2005). Identité professionnelle chez des enseignantes et des enseignants et des enseignantes à statut précaire au secondaire. Dans C. Gervais et L. Portelance (dir.), Des savoirs au coeur de la profession enseignante (p. 207-224). Sherbrooke: Éditions du CRP.

Mushipu Mbombo, D. (2011). De la liberté ou des questions éthiques en éducation aujourd'bui. : Ébauche de réponses par le truchement des modernes. Berne : Peter Lang.

Norberg, K. et Johansson, O. (2007). Ethical dilemmas of Swedish school leaders : Contrasts and common themes. Educational Management Administration Eं Leadership, 35(2), 277-294. http://dx.doi.org/10.1177/1741143207075393

Obin, J.-P. (1996). Les établissements scolaires entre l'éthique et la loi. Paris : Hachette Éducation.

Patenaude, J. (1998). Le dialogue comme paradigme éthique. Réseaux, (83-84), 73-83.

Poirel, E. et Yvon, F. (2012). Les sources de stress, les émotions vécues et les stratégies d'ajustement des directions d'école au Québec. Revue des sciences de l'éducation, 37(3), 595-615. http://dx.doi.org/10.7202/1014759ar

Shapiro, J. P. et Stefkovich, J. A. (2011). Ethical leadership and decision making in education: Applying theoretical perspectives to complex dilemmas ( $3^{\mathrm{e}}$ éd.). New York, NY : Routledge.

Shapiro, J. P., Stefkovich, J. A. et Gutierrez, K. J. (2014). Ethical decision making. Dans C. M. Branson et S. J. Gross (dir.), Handbook of Ethical Educational Leadership (2 éd.) (p. 210-228). New York, NY : Routledge. http://dx.doi.org/10.4324/9780203747582.ch14

St-Vincent, L.-A. (2011a). Dimensions examinées par des novices en enseignement en adaptation scolaire en insertion professionnelle devant un problème éthique à l'école (Thèse de doctorat, Université de Sherbrooke). Repéré à http://savoirs.usherbrooke.ca/handle/11143/964

St-Vincent, L.-A. (2011b). Lorsqu'un problème éthique se présente en période d'insertion professionnelle. Formation et profession, 18(3), 35-37. Repéré à http://www.crifpe.ca/download/verify/1271

St-Vincent, L.-A. (2012). Les dilemmes moraux exprimés par des intervenants au début d'un projet de changement des pratiques à l'école : portrait de trois équipes. McGill Journal of Education / Revue des sciences de l'éducation de McGill, 47(2), 213-230. http://dx.doi.org/10.7202/1013124ar

St-Vincent, L.-A. (2013a). Préparer une direction d'établissement à résoudre un problème éthique rencontré en contexte scolaire. Revue Le Point en administration de l'éducation, 15(3), 16-21. 
St-Vincent, L.-A. (2013b). Développer le cadre de référence éthique des acteurs scolaires pour favoriser la résolution de problèmes éthiques en équipe. Revue suisse de pédagogie spécialisée, (2), 6-12. Repéré à http://saen.ch/sites/default/files/public/docs/1306 rsps st-vincent.pdf

St-Vincent, L.-A. (2013c). La méconnaissance du cadre professionnel chez des enseignantes débutantes québécoises devant un problème éthique à l'école. JCACS. La revue de l'association canadienne pour l'étude de curriculum, 11(1), 136-173.

Tardif, M. (2013). La condition enseignante au Québec du XIXe au XXI siècle : une histoire cousue de fils rouges : précarité, injustice et déclin de l'école publique. Québec, QC : Presses de l'Université Laval.

Trépanier, N. (2005). L’intégration scolaire des élèves en difficulté : une typologie des modèles de service (2éd.). Montréal, QC : Éditions Nouvelles.

Tuana, N. (2014). An ethical leadership developmental framework. Dans C. M. Branson et S. J. Gross (dir.), Handbook of Ethical Educational Leadership (2e éd.) (p. 153-175). New York, NY : Routledge.

http://dx.doi.org/10.4324/9780203747582.ch11

\section{Pour citer cet article}

St-Vincent, L.-A. (2016). Soutien de la direction d'établissement lorsque survient un problème éthique en période d'insertion professionnelle : exploration de quatre avenues. Formation et profession, 24(1), 5-14.

http://dx.doi.org/10.18162/fp.2016.313 